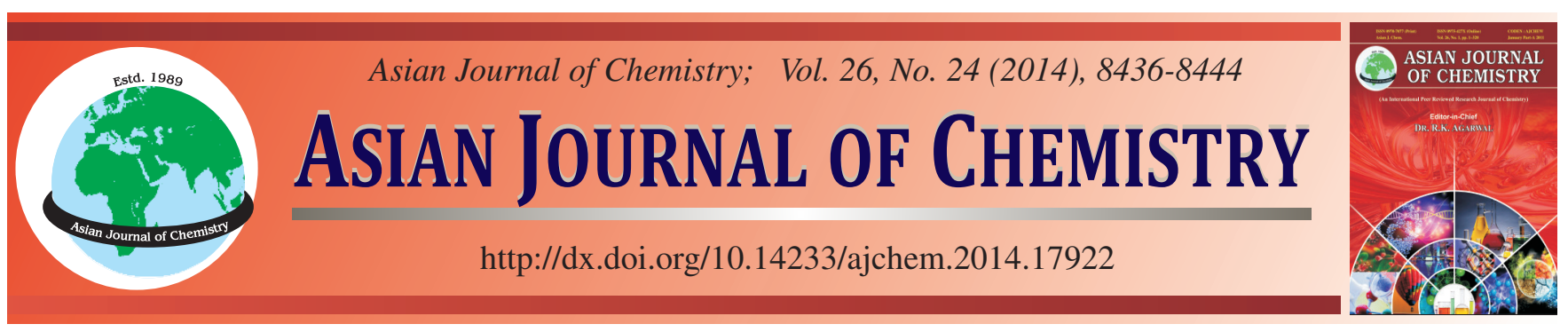

\title{
Antimicrobial and Pharmacological Activities of Some 3-Substituted Thieno[3,2-d]-pyrimidin-4(3H)-one
}

\author{
Aisha S.M. Hossan ${ }^{1, *}$ and Abd El-Galil E. AmR ${ }^{2,3}$
}

${ }^{1}$ Chemistry Department, Girls College of Science, King Khalid University, Abha, Saudi Arabia

${ }^{2}$ Pharmaceutical Chemistry Department, Drug Exploration and Development Chair, College of Pharmacy, King Saud University, Riyadh 11451, Saudi Arabia

${ }^{3}$ Applied Organic Chemistry Department, National Research Center, Cairo, Dokki, Egypt

*Corresponding author: E-mail: hossanaisha@yahoo.com

Received: 30 April 2014;

Accepted: 4 July 2014;

Published online: 1 December 2014;

AJC-16377

\begin{abstract}
A series of new 3-substituted-7-(2-chloro-6-ethoxypyridin-4-yl)-9-(2,4-dichlorophenyl)-2-methylpyrido[3',2':4,5]thieno[3,2- $d]$ pyrimidin4(3H)-one derivatives (3-10) were synthesized using 7-(2-chloro-6-ethoxypyridin-4-yl)-9-(aryl)-2-methyl-4H-pyrido-[3',2':4,5]thieno[3,2$d]$-[1,3]oxazin-4-one (2a,b) as a starting material. All the newly synthesized compounds were evaluated for their antimicrobial activities. The antimicrobial screening showed that many of these obtained compounds have good antimicrobial activities comparable to streptomycin and fusidic acid as reference drugs. Also, the pharmacological screening showed that many compounds have good analgesic and antiparkinsonian activities comparable to Valdecoxib ${ }^{\circledR}$ and Benzotropine ${ }^{\circledR}$ as reference drugs. The structures of newly synthesized compounds were confirmed by IR, ${ }^{1} \mathrm{H}$ NMR, ${ }^{13} \mathrm{C}$ NMR, MS spectral data and elemental analysis.
\end{abstract}

Keywords: Oxazinone, Thienopyrimidine, Schiff base, Imide derivatives, Antimicrobial and pharmacological agents.

\section{INTRODUCTION}

In particular, pyrido[4',3':4,5]thieno[2,3-d]pyrimidin$4(3 H)$-ones were reported to be act as 5 -HT1A receptor antagonists and serotonin reuptake inhibitors ${ }^{1}$, antiinflammatory ${ }^{2,3}$ and antimalarial agents ${ }^{4}$. Moreover, this triheterocyclic system showed highly potent dual 5-HT1A and 5-HT1B antagonists as potential antidepressant drugs ${ }^{5}$. Many of thieno-pyrimidines are found to exhibit a variety of biological activities, including antimicrobial $^{6}$ and analgesic ${ }^{7}$ properties, inhibition of cancer cell proliferation ${ }^{8}$ and antagonism of $\alpha 1$ adre-noceptors ${ }^{9}$. In addition, literature survey reveals that pyrimidines and their synthetic analogs possess a variety of pharmacological activities, such as antiinflammatory, antimicrobial ${ }^{10}$, antimalarial ${ }^{11}$, antituberculous ${ }^{12}$, anti-HIV ${ }^{13}$, antitumor ${ }^{14}$, tyrosine kinase inhibitor $^{15}$, activities. Similarly thienopyrimi-dine $e^{16,17}$ and pyridopyrimidine $^{18}$, the bioisosters of quinazolin-ones are known to possess good anticonvulsant activity. On the other hand, Schiff bases derived from aromatic amines and aromatic aldehydes have a wide variety of applications in many fields, e.g., biological, inorganic and analytical chemistry ${ }^{19,20}$. Also, some of sulfur and nitrogen heterocyclic compounds has a broad spectrum of biological activities i.e. antidepressant ${ }^{21}$, antifungal ${ }^{22}$ activities. In view of these observations and in continuation of our previous works ${ }^{23-32}$ in heterocyclic chemistry, we have herein synthesized some new heterocyclic fused ring systems containing thienopyrimidine moiety and tested their anti-microbial activities in comparison to streptomycin and fusidic acid as positive controls.

\section{EXPERIMENTAL}

Melting points were measured using electrothermal 9100 digital melting point apparatus (Büchi, Switzerland) and are uncorrected. IR spectra were recorded on a Perkin-Elmer 1600 FTIR (Perkin-Elmer) in $\mathrm{KBr}$ discs. ${ }^{1} \mathrm{H}$ - and ${ }^{13} \mathrm{C}$ NMR spectra were measured on a Jeol $5000 \mathrm{MHz}$ spectrometer (Jeol, Japan) in DMSO- $d_{6}$ and chemical shifts were recorded in $\delta \mathrm{ppm}$ relative to the internal standard TMS. The mass spectra were run at $70 \mathrm{eV}$ with a Finnigan SSQ 7000 spectrometer (Madison, WI, USA) using EI and the values of $\mathrm{m} / \mathrm{z}$ are indicated in Dalton. Elemental analyses were performed on a Perkin-Elmer 2400 analyzer (Perkin-Elmer, USA) and were found within \pm $0.4 \%$ of the theoretical values. All reactions were followed by TLC (Silica gel, Aluminum Sheets $60 \mathrm{~F}_{254}$, Merck). Starting materials 2a-c was prepared from the corresponding cinnamoyl derivatives 1a-c according to reported procedure ${ }^{23,24}$.

Synthesis of compounds (3a,b and $4 a, b)$ : A mixture of 7-(2-chloro-6-ethoxypyridin-4-yl)-9-(4-fluorophenyl)-2- 
methyl-4H-pyrido-[3',2':4,5] thieno[3,2- $d][1,3]$ oxazin-4-one $(\mathbf{2 a}, \mathbf{b})(1 \mathrm{mmol})$ and aniline or phenylhydrazine $(1 \mathrm{mmol})$ in glacial acetic acid $(25 \mathrm{~mL})$ was heated under reflux for $6 \mathrm{~h}$. The reaction mixture was concentrated under reduced pressure, pour in ice-cold water and the formed solid was filtered, washed with water, dried and crystallized from the proper solvent to afford the corresponding 3 -substituted pyrido $\left[3^{\prime}, 2^{\prime}: 4,5\right]$ thieno[3,2-d]pyrimidine derivatives $\mathbf{3 a}, \mathbf{b}$ and $\mathbf{4 a}, \mathbf{b}$, respectively.

7-(2-Chloro-6-ethoxypyridin-4-yl)-9-(4-fluorophenyl)2-methyl-3-phenylpyrido $\left[3^{\prime}, 2^{\prime}: 4,5\right]$ thieno-[3,2-d]pyrimidine-4(3H)-one (3a): Yield $85 \%$, m.p. $218-220{ }^{\circ} \mathrm{C}$; IR $\left(\mathrm{KBr}, v_{\max }, \mathrm{cm}^{-1}\right): 1671(\mathrm{C}=\mathrm{O}) ;{ }^{1} \mathrm{H}$ NMR $(500 \mathrm{MHz}, \mathrm{DMSO}-$ $\left.d_{6}, \mathrm{ppm}\right): \delta 1.18\left(\mathrm{t}, 3 \mathrm{H}, \mathrm{CH}_{3}\right), 2.36\left(\mathrm{~s}, 3 \mathrm{H}, \mathrm{CH}_{3}\right), 3.78(\mathrm{q}, 2 \mathrm{H}$, $\left.\mathrm{CH}_{2}\right), 7.12-7.76(\mathrm{~m}, 11 \mathrm{H}, 9 \mathrm{Ph}-\mathrm{H}+2$ pyr-H), 8.54 (s, $1 \mathrm{H}$, pyr5 '-H); ${ }^{13} \mathrm{C}$ NMR (125 MHz, DMSO- $\left.d_{6}, \mathrm{ppm}\right): \delta 14.01,25.12$, $64.18,100.08,101.28,116.24,120.36,121.62,124.35,126.42$, $128.88,129.62,132.75,132.83,136.44,145.66,146.24$, $149.80,151.76,154.12,154.72,157.44,159.65,162.78$, 164.27; MS, $m / z(\%): 543$ [M+1 14], 358 [100, base peak]; Elemental analysis for $\mathrm{C}_{29} \mathrm{H}_{20} \mathrm{~N}_{4} \mathrm{O}_{2} \mathrm{SClF}$ (543.01): Calcd.: C, 64.14; H, 3.71; Cl, 6.53; N, 10.32; S, 5.91; Found: C, 64.10; H, 3.66; Cl, 6.49; N, 10.28; S, 5.86 .

7-(2-Chloro-6-ethoxypyridin-4-yl)-9-(4-chlorophenyl)2-methyl-3-phenylpyrido $\left[3^{\prime}, 2^{\prime}: 4,5\right]$ thieno-[3,2-d]pyrimidine-4(3H)-one (3b): Yield $72 \%$, m.p. $214-216^{\circ} \mathrm{C}$; IR ( $\mathrm{KBr}$, $\left.v_{\max }, \mathrm{cm}^{-1}\right): 1672(\mathrm{C}=\mathrm{O}) ;{ }^{1} \mathrm{H}$ NMR $\left(500 \mathrm{MHz}, \mathrm{DMSO}-d_{6}, \mathrm{ppm}\right)$ : $\delta 1.24\left(\mathrm{t}, 3 \mathrm{H}, \mathrm{CH}_{3}\right), 2.24\left(\mathrm{~s}, 3 \mathrm{H}, \mathrm{CH}_{3}\right), 3.82\left(\mathrm{q}, 2 \mathrm{H}, \mathrm{CH}_{2}\right)$, 7.08-7.75 (m, 11H, 9 Ph-H + 2 pyr-H), 8.62 (s, 1H, pyr-5'-H); ${ }^{13} \mathrm{C}$ NMR (125 MHz, DMSO- $\left.d_{6}, \mathrm{ppm}\right): \delta 13.82,26.33,64.12$, $100.01,101.12,120.50,121.72,124.32,126.82,128.01$, $128.84,129.15,132.82,133.40,135.80,136.43,145.38$, $146.97,149.75,150.82,153.80,154.62,157.32,160.00$, 164.20; MS, m/z (\%): 559 [ $\left.\mathrm{M}^{+}, 8\right], 132$ [100, base peak]; Elemental analysis for $\mathrm{C}_{29} \mathrm{H}_{20} \mathrm{~N}_{4} \mathrm{O}_{2} \mathrm{SCl}_{2}$ (559.47): Calcd.: C, 62.26; H, 3.60; Cl, 12.67; N, 10.01; S, 5.73; Found: C, 62.20; $\mathrm{H}, 3.55 ; \mathrm{Cl}, 12.60 ; \mathrm{N}, 9.95 ; \mathrm{S}, 5.65$.

7-(2-Chloro-6-ethoxypyridin-4-yl)-9-(4-fluorophenyl)3-benzyl-2-methylpyrido $\left[3^{\prime}, 2^{\prime}: 4,5\right]$ thieno- $[3,2-d]$ pyrimidine-4(3H)-one (4a): Yield $65 \%$, m.p. $168-170{ }^{\circ} \mathrm{C}$; IR ( $\mathrm{KBr}$, $\left.\mathrm{v}_{\max }, \mathrm{cm}^{-1}\right): 3348(\mathrm{NH}), 1675(\mathrm{C}=\mathrm{O}) ;{ }^{1} \mathrm{H}$ NMR $(500 \mathrm{MHz}$, DMSO- $\left.d_{6}, \mathrm{ppm}\right): \delta 1.30$ (t, $\left.3 \mathrm{H}, \mathrm{CH}_{3}\right), 2.34\left(\mathrm{~s}, 3 \mathrm{H}, \mathrm{CH}_{3}\right), 3.84$ (q, $\left.2 \mathrm{H}, \mathrm{CH}_{2}\right), 6.68-7.71(\mathrm{~m}, 11 \mathrm{H}, 9 \mathrm{Ph}-\mathrm{H}+2 \mathrm{pyr}-\mathrm{H}), 8.54$ (s, $1 \mathrm{H}$, pyr-5'-H), 9.15 (s, $1 \mathrm{H}, \mathrm{NH}$ changeable with $\left.\mathrm{D}_{2} \mathrm{O}\right) ;{ }^{13} \mathrm{C}$ NMR (125 MHz, DMSO- $\left.d_{6}, \mathrm{ppm}\right): \delta$ 13.78, 24.96, 64.18, $101.00,102.42,113.24,116.24,119.34,121.02,126.42$, $129.04,129.86,132.91,136.54,145.68,146.32,150.12$, $151.05,151.96,154.11,154.65,157.22,159.56,162.78$, 164.35; MS, $m / z(\%): 559$ [ $\left.\mathrm{M}^{+}+1,12\right], 234$ [100, base peak]. Elemental analysis for $\mathrm{C}_{29} \mathrm{H}_{21} \mathrm{~N}_{5} \mathrm{O}_{2} \mathrm{SClF}$ (558.03): Calcd.: $\mathrm{C}$, 62.42; H, 3.79; Cl, 6.35; N, 12.55; S, 5.75; Found: C, 62.38; $\mathrm{H}, 3.75 ; \mathrm{Cl}, 6.30 ; \mathrm{N}, 12.50 ; \mathrm{S}, 5.70$.

7-(2-Chloro-6-ethoxypyridin-4-yl)-9-(4-chlorophenyl)3-benzyl-2-methylpyrido $\left[3^{\prime}, 2^{\prime}: 4,5\right]$ thieno- $[3,2-d]$ pyrimidine-4(3H)-one (4b): Yield $56 \%$, m.p. $206-208^{\circ} \mathrm{C}$; IR (KBr, $\left.\mathrm{v}_{\max }, \mathrm{cm}^{-1}\right): 3354(\mathrm{NH}), 1671(\mathrm{C}=\mathrm{O}) ;{ }^{1} \mathrm{H}$ NMR $(500 \mathrm{MHz}$, DMSO- $\left.d_{6}, \mathrm{ppm}\right): \delta 1.40\left(\mathrm{t}, 3 \mathrm{H}, \mathrm{CH}_{3}\right), 2.34\left(\mathrm{~s}, 3 \mathrm{H}, \mathrm{CH}_{3}\right), 3.92$ (q, 2H, $\left.\mathrm{CH}_{2}\right), 6.72-7.74(\mathrm{~m}, 11 \mathrm{H}, 9 \mathrm{Ph}-\mathrm{H}+2 \mathrm{pyr}-\mathrm{H}), 8.68$ (s, $1 \mathrm{H}$, pyr-5'-H), 9.08 (s, $1 \mathrm{H}, \mathrm{NH}$ changeable with $\left.\mathrm{D}_{2} \mathrm{O}\right) ;{ }^{13} \mathrm{C}$ NMR (125 MHz, DMSO- $\left.d_{6}, \mathrm{ppm}\right): \delta$ 14.02, 27.05, 64.22,
$99.88,100.25,113.25,119.65,121.15,127.31,128.01,129.12$, $129.55,133.56,136.18,136.84,145.37,146.82,149.66$, 151.06, 151.14, 153.88, 155.00, 157.52, 159.86, 164.16; MS, $\mathrm{m} / \mathrm{z}(\%): 574\left[\mathrm{M}^{+}, 5\right], 412$ [100, base peak]. Elemental analysis for $\mathrm{C}_{29} \mathrm{H}_{21} \mathrm{~N}_{5} \mathrm{O}_{2} \mathrm{SCl}_{2}$ (574.48): Calcd.: C, 60.63; H, 3.68; Cl, 12.34; N, 12.19; S, 5.58; Found: C, 60.55; H, 3.62; Cl, 12.30; N, 12.14; S, 5.54 .

Synthesis of 3-amino-7-(2-chloro-6-ethoxypyridin-4yl)-9-aryl-2-methylpyrido[3',2':4,5]-thieno[3,2-d]pyrimidin-4(3H)-one $(5 \mathbf{a}, \mathbf{b})$ : A mixture of $\mathbf{2 a , b}(1 \mathrm{mmol})$ and hydrazine hydrate $(8 \mathrm{mmol})$ in absolute ethanol $(50 \mathrm{~mL})$ was refluxed for $4 \mathrm{~h}$. After cooling, the obtained solid was collected by filtration, dried and crystallized from the proper solvent to give the title compound derivatives $\mathbf{5 a}, \mathbf{b}$, respectively.

3-Amino-7-(2-chloro-6-ethoxypyridin-4-yl)-9-(4fluorophenyl)-2-methylpyrido $\left[3^{\prime}, 2^{\prime}: 4,5\right]$ thieno $[3,2-$ d]pyrimidin-4(3H)-one (5a): Yield $68 \%$, m.p. $268-270{ }^{\circ} \mathrm{C}$; IR $\left(\mathrm{KBr}, \mathrm{v}_{\max }, \mathrm{cm}^{-1}\right)$ : $3365\left(\mathrm{NH}_{2}\right), 1672(\mathrm{C}=\mathrm{O}) ;{ }^{1} \mathrm{H}$ NMR (500 MHz, DMSO- $\left.d_{6}, \mathrm{ppm}\right): \delta 1.24\left(\mathrm{t}, 3 \mathrm{H}, \mathrm{CH}_{3}\right), 2.34\left(\mathrm{~s}, 3 \mathrm{H}, \mathrm{CH}_{3}\right)$, 3.86 (q, 2H, $\left.\mathrm{CH}_{2}\right), 4.78$ (s, 2H, $\mathrm{NH}_{2}$ exchangeable with $\left.\mathrm{D}_{2} \mathrm{O}\right)$, 7.05-7.74 (m, 6H, 4 Ph-H + 2 pyr-H), 8.74 (s, 1H, pyr-5'-H); ${ }^{13} \mathrm{C}$ NMR (125 MHz, DMSO- $\left.d_{6}, \mathrm{ppm}\right): \delta 14.01,24.99,64.22$, 100.16, 101.41, 116.19, 120.32, 126.44, 128.98, 132.91, 136.40, 145.77, 146.22, 149.80, 151.76, 154.10, 154.86, 157.30, 159.60, 162.70, 164.28; MS, m/z (\%): $482\left[\mathrm{M}^{+}, 24\right], 136$ [100, base peak]. Elemental analysis for $\mathrm{C}_{23} \mathrm{H}_{17} \mathrm{~N}_{5} \mathrm{O}_{2} \mathrm{SClF}$ (481.93): Calcd.: C, 57.32; H, 3.56; Cl, 7.36; N, 14.53; S, 6.65; Found: C, 57.28; $\mathrm{H}, 3.50 ; \mathrm{Cl}, 7.30 ; \mathrm{N}, 14.48 ; \mathrm{S}, 6.60$.

3-Amino-7-(2-chloro-6-ethoxypyridin-4-yl)-9-(4chlorophenyl) -2-methylpyrido $\left[3^{\prime}, 2^{\prime}: 4,5\right]$ thieno $[3,2-$ d]pyrimidin-4(3H)-one (5b): Yield $75 \%$, m.p. 216-218 ${ }^{\circ} \mathrm{C}$; IR $\left(\mathrm{KBr}, \mathrm{v}_{\max }, \mathrm{cm}^{-1}\right)$ : $3358\left(\mathrm{NH}_{2}\right), 1673(\mathrm{C}=\mathrm{O}) ;{ }^{1} \mathrm{H}$ NMR $(500$ $\left.\mathrm{MHz}, \mathrm{DMSO}-d_{6}, \mathrm{ppm}\right): \delta 1.30\left(\mathrm{t}, 3 \mathrm{H}, \mathrm{CH}_{3}\right), 2.26\left(\mathrm{~s}, 3 \mathrm{H}, \mathrm{CH}_{3}\right)$, 3.80 (q, $\left.2 \mathrm{H}, \mathrm{CH}_{2}\right), 4.84$ (s, $2 \mathrm{H}, \mathrm{NH}_{2}$ exchangeable with $\mathrm{D}_{2} \mathrm{O}$ ), 7.18-7.78 (m, 6H, 4 Ph-H + 2 pyr-H), 8.77 (s, 1H, pyr-5'-H); ${ }^{13} \mathrm{C}$ NMR (125 MHz, DMSO- $\left.d_{6}, \mathrm{ppm}\right): \delta 13.98,24.97,64.22$, 100.12 , 101.48, 120.36, 126.45, 128.22, 128.98, 134.55, $135.76,136.37,145.76,146.26,149.75,151.70,154.09$, 154.85, 157.32, 159.64, 164.32; MS, $m / z(\%): 498\left[\mathrm{M}^{+}, 24\right]$, 164 [100, base peak]. Elemental analysis for $\mathrm{C}_{23} \mathrm{H}_{17} \mathrm{~N}_{5} \mathrm{O}_{2} \mathrm{SCl}_{2}$ (498.38): Calcd.: C, 55.43; H, 3.44; Cl, 14.23; N, 14.05; S, 6.43; Found: C, 55.38; H, 3.40; Cl, 14.18; N, 14.00; S, 6.38 .

Synthesis of 1-\{(2-Chloro-6-ethoxypyridin-4-yl)-9aryl-2-methyl-4-oxopyrido $\left[2^{\prime}, 3^{\prime}: 4,5\right]$ thieno $[3,2-d]$ pyrimidin-3(4H)-yl\}-3-phenylthiourea $(6 \mathbf{6}, \mathbf{b})$ : To a mixture of 5a,b $(1 \mathrm{mmol})$ and phenylisothiocyanate $(1 \mathrm{mmol})$ in dry dioxane $(25 \mathrm{~mL})$, triethylamine $(2 \mathrm{~mL})$ was added with stirring. The reaction mixture was heated under reflux for $10 \mathrm{~h}$. The solvent was evaporated under reduced pressure, the obtained residue was solidified with $n$-hexane. The solid formed was collected by filtration, washed with diethyl ether, dried and crystallized from the proper solvents to afford the corresponding thiourea derivatives $\mathbf{6} \mathbf{a}, \mathbf{b}$, respectively.

1-\{(2-Chloro-6-ethoxypyridin-4-yl)-9-(4-fluorophenyl)2-methyl-4-oxopyrido $\left[2^{\prime}, 3^{\prime}: 4,5\right]$ thieno $[3,2-d]$ pyrimidin3(4H)-yl\}-3-phenylthiourea (6a): Yield 72\%, m.p. 156-158 ${ }^{\circ} \mathrm{C}$; IR $\left(\mathrm{KBr}, v_{\max }, \mathrm{cm}^{-1}\right): 3338-3250(\mathrm{NH}), 1678(\mathrm{C}=\mathrm{O}), 1236$ $(\mathrm{C}=\mathrm{S}) ;{ }^{1} \mathrm{H}$ NMR $\left(500 \mathrm{MHz}, \mathrm{DMSO}-d_{6}, \mathrm{ppm}\right): \boldsymbol{\delta} 1.16$ (s, $3 \mathrm{H}$, $\left.\mathrm{CH}_{3}\right), 2.14\left(\mathrm{t}, 3 \mathrm{H}, \mathrm{CH}_{3}\right), 3.86\left(\mathrm{q}, 2 \mathrm{H}, \mathrm{CH}_{2}\right), 4.28,4.36(2 \mathrm{~s}, 2 \mathrm{H}$, 
2NH exchangeable with $\left.\mathrm{D}_{2} \mathrm{O}\right), 6.95-7.78$ ( $\mathrm{m}, 11 \mathrm{H}, 9 \mathrm{Ph}-\mathrm{H}+2$ pyr-H), 8.47 (s, $1 \mathrm{H}$, pyr- $\left.5^{\prime}-\mathrm{H}\right) ;{ }^{13} \mathrm{C}$ NMR (125 MHz, DMSO$\left.d_{6}, \mathrm{ppm}\right): \delta 13.88,27.12,64.20,99.90,100.28,113.25,119.60$, $121.13,127.30,128.05,129.17,129.56,133.58,136.22,136.90$, $145.40,146.86,149.68,151.00,151.08,153.90,155.04$, 157.55, 159.80, 164.20, 177.66; MS, $m / z(\%): 617\left[\mathrm{M}^{+}, 12\right]$, 150 [100, base peak]. Elemental analysis for $\mathrm{C}_{30} \mathrm{H}_{22} \mathrm{~N}_{6} \mathrm{O}_{2} \mathrm{~S}_{2} \mathrm{ClF}$ (617.12): Calcd.: C, 58.39; H, 3.59; Cl, 5.74; N, 13.62; S, 10.39; Found: C, 58.33; H, 3.54; Cl, 5.70; N, 13.58; S, 10.34 .

1-\{(2-Chloro-6-ethoxypyridin-4-yl)-9-(4-chlorophenyl)2-methyl-4-oxopyrido $\left[2^{\prime}, 3^{\prime}: 4,5\right]$ thieno[3,2-d]pyrimidin3(4H)-yl\}-3-phenylthiourea (6b): Yield 78 \%, m.p. 216-218 ${ }^{\circ} \mathrm{C}$; IR $\left(\mathrm{KBr}, v_{\max }, \mathrm{cm}^{-1}\right): 3348-3232(\mathrm{NH}), 1676(\mathrm{C}=\mathrm{O}), 1242$ $(\mathrm{C}=\mathrm{S}) ;{ }^{1} \mathrm{H}$ NMR $\left(500 \mathrm{MHz}, \mathrm{DMSO}-d_{6}, \mathrm{ppm}\right): \boldsymbol{\delta} 1.18$ (s, 3H, $\left.\mathrm{CH}_{3}\right), 2.18$ (t, 3H, $\left.\mathrm{CH}_{3}\right), 3.79$ (q, 2H, $\left.\mathrm{CH}_{2}\right), 4.32,4.42(2 \mathrm{~s}, 2 \mathrm{H}$, $2 \mathrm{NH}$ exchangeable with $\left.\mathrm{D}_{2} \mathrm{O}\right), 6.98-7.85(\mathrm{~m}, 11 \mathrm{H}, 9 \mathrm{Ph}-\mathrm{H}+2$ pyr-H), 8.56 (s, 1H, pyr-5'-H); ${ }^{13} \mathrm{C}$ NMR (125 MHz, DMSO$\left.d_{6}, \mathrm{ppm}\right): \delta 14.05,27.15,64.20,99.92,100.26,113.28,119.60$, $121.22,127.24,128.11,129.22,129.56,133.58,136.22$, $136.85,145.40,146.85,149.68,151.13,151.10,153.89$, $155.02,157.50,159.80,164.14,177.60 ; \mathrm{MS}, \mathrm{m} / \mathrm{z}(\%): 634$ $\left[\mathrm{M}^{+}, 32\right], 136$ [100, base peak]. Elemental analysis for $\mathrm{C}_{30} \mathrm{H}_{22} \mathrm{~N}_{6} \mathrm{O}_{2} \mathrm{~S}_{2} \mathrm{Cl}_{2}$ (633.57): Calcd.: C, 56.87; H, 3.50; Cl, 11.19; N, 13.26; S, 10.12; Found: C, 56.82; H, 3.45; Cl, 11.13; N, 13.20; S, 10.08 .

2-Chloro-6-ethoxy-4-[2-methyl-4-oxo-3-(phthalimido or 2,3,4,5-tetrachlorophthalimido)-9-aryl-3,4-dihydropyrido[3',2':4,5] thieno[3,2-d]pyrimidin-7-yl]pyridine 7a-d: A mixture of $\mathbf{5 a , b}(1 \mathrm{mmol})$ and phthalic anhydride or 2,3,4,5tetrachlorophthalic anhydride $(1 \mathrm{mmol})$ in glacial acetic acid $(30 \mathrm{~mL})$ was heated under reflux for $6 \mathrm{~h}$. The reaction mixture was evaporated under reduced pressure, the obtained residue was solidified with diethyl ether. The obtained solid was filtered off, washed with diethyl ether, dried and crystallized from the proper solvents to afford the corresponding N-phthalimido derivatives $7 \mathbf{a}-\mathbf{d}$, respectively.

2-Chloro-6-ethoxy-4-[2-methyl-4-oxo-3-(phthalimido)9-(4-fluorophenyl)-3,4-dihydropyrido-[3',2':4,5] thieno[3,2d]pyrimidin-7-yl]pyridine (7a): Yield 70 \%, m.p. 224-226 ${ }^{\circ} \mathrm{C}$; IR $\left(\mathrm{KBr}, \mathrm{v}_{\max }, \mathrm{cm}^{-1}\right): 1722(\mathrm{C}=\mathrm{O}), 1670(\mathrm{C}=\mathrm{O}) ;{ }^{1} \mathrm{H}$ NMR $(500$ MHz, DMSO- $\left.d_{6}, \mathrm{ppm}\right): \delta 1.28\left(\mathrm{t}, 3 \mathrm{H}, \mathrm{CH}_{3}\right), 2.35\left(\mathrm{~s}, 3 \mathrm{H}, \mathrm{CH}_{3}\right)$, 3.82 (q, 2H, $\left.\mathrm{CH}_{2}\right), 6.70-7.56$ (m, 6H, $4 \mathrm{Ph}-\mathrm{H}+2$ pyr-H), 7.868.04 (m, 4H, Ar-H), 8.48 (s, 1H, pyr-5'-H); ${ }^{13} \mathrm{C}$ NMR $(125 \mathrm{MHz}$, DMSO- $\left.d_{6}, \mathrm{ppm}\right): \delta 13.95,25.00,64.24,100.18,101.42$, $116.22,120.36,123.18,126.45,128.96,131.78,132.45$, $132.90,136.42,145.75,146.18,149.84,151.78,154.12$, $154.85,157.32,159.65,162.760,164.30,165.15 ; \mathrm{MS}, \mathrm{m} / z$ (\%): 612 [ $\left.\mathrm{M}^{+}, 12\right], 254$ [100, base peak]. Elemental analysis for $\mathrm{C}_{31} \mathrm{H}_{19} \mathrm{~N}_{5} \mathrm{O}_{4} \mathrm{SClF}$ (612.03): Calcd.: C, 60.84; H, 3.13; Cl, 5.79; N, 11.44; S, 5.24; Found: C, 60.80; H, 3.08; Cl, 5.74; N, $11.40 ;$ S, 5.20 .

2-Chloro-6-ethoxy-4-[2-methyl-4-oxo-3-(phthalimido)9-(4-chlorophenyl)-3,4-dihydropyrido-[3',2':4,5] thieno[3,2d]pyrimidin-7-yl]pyridine (7b): Yield $65 \%$, m.p. 178-180 ${ }^{\circ} \mathrm{C}$; IR $\left(\mathrm{KBr}, \mathrm{v}_{\max }, \mathrm{cm}^{-1}\right)$ : $1726(\mathrm{C}=\mathrm{O}), 1668(\mathrm{C}=\mathrm{O}) ;{ }^{1} \mathrm{H}$ NMR (500 MHz, DMSO- $\left.d_{6}, \mathrm{ppm}\right): \delta 1.32\left(\mathrm{t}, 3 \mathrm{H}, \mathrm{CH}_{3}\right), 2.30(\mathrm{~s}, 3 \mathrm{H}$, $\mathrm{CH}_{3}$ ), 3.78 (q, 2H, $\mathrm{CH}_{2}$ ), 6.85-7.64 (m, 6H, $4 \mathrm{Ph}-\mathrm{H}+2$ pyr$\mathrm{H}), 7.82-8.10(\mathrm{~m}, 4 \mathrm{H}, \mathrm{Ar}-\mathrm{H}), 8.52\left(\mathrm{~s}, 1 \mathrm{H}, \mathrm{pyr}-5^{\prime}-\mathrm{H}\right) ;{ }^{13} \mathrm{C}$ NMR (125 MHz, DMSO- $\left.d_{6}, \mathrm{ppm}\right): \delta 14.12,24.95,64.20,100.24$,
$101.52,120.38,123.24,126.40,128.20,128.93,131.80$, $132.52,134.60,135.72,136.35,145.70,146.23,149.70$, 151.65, 154.00, 154.80, 157.30, 159.60, 164.30, 164.80; MS, $\mathrm{m} / \mathrm{z}(\%): 429\left[\mathrm{M}^{+}+1,12\right], 318$ [100, base peak]. Elemental analysis for $\mathrm{C}_{31} \mathrm{H}_{19} \mathrm{~N}_{5} \mathrm{O}_{4} \mathrm{SCl}_{2}$ (628.48): Calcd.: C, 59.24; $\mathrm{H}$, 3.05; Cl, 11.28; N, 11.14; S, 5.10; Found: C, 59.20; H, 3.00; $\mathrm{Cl}, 11.23 ; \mathrm{N}, 11.10 ; \mathrm{S}, 5.04$.

2-Chloro-6-ethoxy-4-[2-methyl-4-oxo-3-(2,3,4,5-tetrachlorophthalimido)-9-(4-fluorophenyl)-3,4-di-hydropyrido $\left[3^{\prime}, 2^{\prime}: 4,5\right]$ thieno[3,2- $\left.d\right]$ pyrimidin-7-yl]pyridine (7c): Yield $65 \%$, m.p. $115-117^{\circ} \mathrm{C}$; IR (KBr, $\left.v_{\max }, \mathrm{cm}^{-1}\right)$ : $1732(\mathrm{C}=\mathrm{O})$, $1674(\mathrm{C}=\mathrm{O}) ;{ }^{1} \mathrm{H}$ NMR $\left(500 \mathrm{MHz}, \mathrm{DMSO}-d_{6}, \mathrm{ppm}\right): \delta 1.32(\mathrm{t}$, $\left.3 \mathrm{H}, \mathrm{CH}_{3}\right), 2.32$ (s, 3H, $\mathrm{CH}_{3}$ ), 3.86 (q, 2H, $\left.\mathrm{CH}_{2}\right), 6.76-7.62$ (m, $6 \mathrm{H}, 4 \mathrm{Ph}-\mathrm{H}+2$ pyr-H), 8.54 (s, 1H, pyr-5'-H); ${ }^{13} \mathrm{C}$ NMR (125 MHz, DMSO- $\left.d_{6}, \mathrm{ppm}\right): \delta 13.98,25.02,64.30,100.16,101.45$, $116.25,120.34,126.48,127.18,128.98,131.75,132.92$, $134.75,136.46,145.78,146.25,149.85,151.75,154.16$, $154.87,157.34,159.60,162.70,164.32,165.18 ; \mathrm{MS}, \mathrm{m} / \mathrm{z}(\%)$ : $750\left[\mathrm{M}^{+}, 6\right], 280$ [100, base peak]. Elemental analysis for $\mathrm{C}_{31} \mathrm{H}_{15} \mathrm{~N}_{5} \mathrm{O}_{4} \mathrm{SCl}_{5} \mathrm{~F}$ (749.81): Calcd.: C, 49.66; H, 2.02; Cl, 23.64; N, 9.34; S, 4.28; Found: C, 49.60; H, 1.95; Cl, 23.60; N, 9.29; S, 4.21.

2-Chloro-6-ethoxy-4-[2-methyl-4-oxo-3-(2,3,4,5tetrachlorophthalimido)-9-(4-chlorophenyl)-3,4-di-hydropyrido $\left[3^{\prime}, 2^{\prime}: 4,5\right]$ thieno[3,2-d]pyrimidin-7-yl]pyridine (7d): Yield $76 \%$, m.p. $235-237^{\circ} \mathrm{C}$; IR (KBr, $\left.v_{\max }, \mathrm{cm}^{-1}\right)$ : $1737(\mathrm{C}=\mathrm{O})$, $1672(\mathrm{C}=\mathrm{O}) ;{ }^{1} \mathrm{H}$ NMR $\left(500 \mathrm{MHz}, \mathrm{DMSO}-d_{6}, \mathrm{ppm}\right): \delta 1.24(\mathrm{t}$, $\left.3 \mathrm{H}, \mathrm{CH}_{3}\right), 2.36$ (s, 3H, $\left.\mathrm{CH}_{3}\right), 3.83$ (q, 2H, $\left.\mathrm{CH}_{2}\right), 6.88-7.65$ (m, $6 \mathrm{H}, 4 \mathrm{Ph}-\mathrm{H}+2$ pyr-H), 8.48 (s, 1H, pyr-5'-H); ${ }^{13} \mathrm{C} \mathrm{NMR} \mathrm{(125}$ MHz, DMSO- $\left.d_{6}, \mathrm{ppm}\right): \delta 13.98,24.90,64.24,100.28,101.56$, $120.42,126.45,127.16,128.24,128.90,132.94,134.66,134.70$, 135.70, 136.42, 145.74, 146.28, 149.77, 151.69, 154.05, 154.82, 157.34, 159.65, 164.35, 164.84; MS, $m / z(\%): 766\left[\mathrm{M}^{+}, 16\right]$, 450 [100, base peak]. Elemental analysis for $\mathrm{C}_{31} \mathrm{H}_{15} \mathrm{~N}_{5} \mathrm{O}_{4} \mathrm{SCl}_{6}$ (766.26): Calcd.: C, 48.59; H, 1.97; Cl, 27.76; N, 9.14; S, 4.18; Found: C, 48.54; H, 1.90; Cl, 27.70; N, 9.10; S, 4.12.

2-Chloro-6-ethoxy-4-[2-methyl-4-oxo-3-(4-fluorophenylmethylidene)amino)-9-aryl-3,4-dihydropyrido$\left[3^{\prime}, 2^{\prime}: 4,5\right]$ thieno[3,2-d]pyrimidin-7-yl]pyridines (8a,b): A mixture of $\mathbf{5 a}, \mathbf{b}(1 \mathrm{mmol})$ and 4-fluorobenzaldehyde (1 mmol) in absolute ethanol $(20 \mathrm{~mL})$ was refluxed for $5 \mathrm{~h}$. After cooling, the obtained solid was filtered off, washed with ethanol, dried and and crystallized from the proper solvents to give the corresponding the title compounds $\mathbf{8 a}, \mathbf{b}$, respectively.

2-Chloro-6-ethoxy-4-[2-methyl-4-oxo-3-(4-fluorophenylmethylidene)amino)-9-(4-fluorophenyl)-3,4dihydropyrido $\left[3^{\prime}, 2^{\prime}: 4,5\right]$ thieno $[3,2-d]$ pyrimidin-7yl]pyridine (8a): Yield $66 \%$, m.p. $135-137^{\circ} \mathrm{C}$; IR (KBr, $v_{\max }$, $\left.\mathrm{cm}^{-1}\right): 1675(\mathrm{C}=\mathrm{O}) ;{ }^{1} \mathrm{H}$ NMR $\left(500 \mathrm{MHz}, \mathrm{DMSO}-d_{6}, \mathrm{ppm}\right): \delta$ 1.18 (t, 3H, $\mathrm{CH}_{3}$ ), 2.32 (s, 3H, $\left.\mathrm{CH}_{3}\right), 3.78$ (q, 2H, $\left.\mathrm{CH}_{2}\right), 6.25$ (s, $1 \mathrm{H}, \mathrm{CH}=\mathrm{N}), 6.85-7.85$ (m, 10H, $8 \mathrm{Ph}-\mathrm{H}+2 \mathrm{pyr}-\mathrm{H}), 8.52$ (s, 1H, pyr-5'-H); ${ }^{13} \mathrm{C}$ NMR (125 MHz, DMSO- $\left.d_{6}, \mathrm{ppm}\right): \delta$ $14.08,25.10,64.32,100.22,101.36,115.45,116.28,120.45$, $123.16,126.52,130.15,130.70,132.12,136.48,143.18$, $145.82,146.22,149.86,152.15,154.10,155.05,157.28$, 159.72, 162.74, 164.90, 166.75; MS, $m / z$ (\%): 588 [M+, 5], 122 [100, base peak]. Elemental analysis for $\mathrm{C}_{30} \mathrm{H}_{20} \mathrm{~N}_{5} \mathrm{O}_{2} \mathrm{SClF}_{2}$ (588.03): Calcd.: C, 61.28; H, 3.43; Cl, 6.03; N, 11.91; S, 5.45; Found: C, 61.20; H, 3.38; Cl, 5; .96N, 11.85; S, 5.40. 
2-Chloro-6-ethoxy-4-[2-methyl-4-oxo-3-(4-chlorophenylmethylidene)amino)-9-(4-fluorophenyl)-3,4dihydropyrido $\left[3^{\prime}, 2^{\prime}: 4,5\right]$ thieno $[3,2-d]$ pyrimidin-7yl]pyridine (8b): Yield $78 \%$, m.p. $214-216^{\circ} \mathrm{C}$; IR (KBr, $v_{\max }$, $\left.\mathrm{cm}^{-1}\right): 1678(\mathrm{C}=\mathrm{O}) ;{ }^{1} \mathrm{H}$ NMR $\left(500 \mathrm{MHz}, \mathrm{DMSO}-d_{6}, \mathrm{ppm}\right): \delta$ 1.22 (t, 3H, $\left.\mathrm{CH}_{3}\right), 2.36$ (s, 3H, $\left.\mathrm{CH}_{3}\right), 3.75$ (q, $\left.2 \mathrm{H}, \mathrm{CH}_{2}\right), 6.12$ (s, 1H, CH=N), 6.92-7.95 (m, 10H, 8 Ph-H + 2 pyr-H), 8.54 (s, $\left.1 \mathrm{H}, \mathrm{pyr}-5^{\prime}-\mathrm{H}\right) ;{ }^{13} \mathrm{C}$ NMR (125 MHz, DMSO-d $\left.d_{6}, \mathrm{ppm}\right): \delta$ $14.45,24.90,64.28,100.02,101.50,116.08,120.44,123.32$, $126.42,128.90,130.80,131.52,134.56,135.70,136.38$, $144.08,145.72,146.20,149.66,151.68,154.08,154.76$, 157.34, 159.62, 165.04, 166.05; MS, $m / z(\%): 604\left[\mathrm{M}^{+}, 16\right]$, 287 [100, base peak]. Elemental analysis for $\mathrm{C}_{30} \mathrm{H}_{20} \mathrm{~N}_{5} \mathrm{O}_{2} \mathrm{SCl}_{2} \mathrm{~F}$ (604.48): Calcd.: C, 59.61; H, 3.33; Cl, 3.14; N, 11.59; S, 5.30; Found: C, 59.55; H, 3.28; Cl, 11.70; N, 11.54; S, 5.25.

\section{Synthesis of compounds 9a,b and 10a,b}

A mixture of $\mathbf{5 a}, \mathbf{b}(2 \mathrm{mmol})$ and tetracarboxylic acid dianhydride, namely, 1,2,4,5-benzene tetracarboxylic dianhydride or 1,8,4,5-naphthalenetetracarboxylic dianhydride $(1 \mathrm{mmol})$ in glacial acetic acid $(30 \mathrm{~mL})$ was heated under reflux for $5 \mathrm{~h}$. The obtained residue was filtered off, washed with acetic acid, dried and crystallized from the proper solvents to afford the corresponding bis-derivatives $\mathbf{9 a}, \mathbf{b}$ and $\mathbf{1 0 a}, \mathbf{b}$, respectively.

2,6-Bis[7-(2-chloro-6-ethoxypyridin-4-yl)-9-(4-fluorophenyl)-3-hydroxy-2-methylpyrido[3',2':4,5]-thieno[3,2d]pyrimidin-4(3H)-on-3-yl)]pyrrolo[3,4-f]isoindole$\mathbf{1 , 3 , 5 , 7}(\mathbf{2 H}, \mathbf{6 H})$-tetrone (9a): Yield $55 \%$, m.p. $278-280{ }^{\circ} \mathrm{C}$; IR $\left(\mathrm{KBr}, v_{\max }, \mathrm{cm}^{-1}\right): 1756(\mathrm{C}=\mathrm{O}), 1672(\mathrm{C}=\mathrm{O}) ;{ }^{1} \mathrm{H}$ NMR $(500$ MHz, DMSO- $\left.d_{6}, \mathrm{ppm}\right): \delta 1.32\left(\mathrm{t}, 6 \mathrm{H}, 2 \mathrm{CH}_{3}\right), 2.42(\mathrm{~s}, 6 \mathrm{H}, 2$ $\left.\mathrm{CH}_{3}\right), 3.86$ (q, 4H, $\left.2 \mathrm{CH}_{2}\right), 6.80-7.92$ (m, 12H, $8 \mathrm{Ph}-\mathrm{H}+4$ pyr$\mathrm{H}), 8.56$ (s, 2H, 2 pyr-5'-H), 9.15 (s, 2H, benzene-H); ${ }^{13} \mathrm{C}$ NMR (125 MHz, DMSO- $\left.d_{6}, \mathrm{ppm}\right): \delta 13.88,25.01,64.20,101.04$, $101.68,116.32,120.38,123.08,125.45,128.90,131.73,135.16$, 136.40, 145.70, 146.24, 149.82, 151.80, 154.16, 154.80, 157.30, 159.60, 162.76, 164.22, 165.34; MS, $m / z$ (\%): 1146 [M+, 4], 465 [100, base peak]. Elemental analysis for $\mathrm{C}_{56} \mathrm{H}_{32} \mathrm{~N}_{10} \mathrm{O}_{8} \mathrm{~S}_{2} \mathrm{Cl}_{2} \mathrm{~F}_{2}$ (1145.95): Calcd.: C, 58.69; H, 2.81; Cl, 6.19; N, 12.22; S, 5.60; Found: C, 58.65; H, 2.77; Cl, 6.15; N, 12.17; S, 5.54.

2,6-Bis[7-(2-chloro-6-ethoxypyridin-4-yl)-9-(4-chlorophenyl)-3-hydroxy-2-methylpyrido $\left[3^{\prime}, 2^{\prime}: 4,5\right]$-thieno[3,2$d$ ]pyrimidin-4(3H)-on-3-yl)]pyrrolo[3,4-f]isoindole1,3,5,7(2H,6H)-tetrone (9b): Yield $62 \%$, m.p. 302-304 ${ }^{\circ} \mathrm{C}$; IR $\left(\mathrm{KBr}, v_{\max }, \mathrm{cm}^{-1}\right): 1748(\mathrm{C}=\mathrm{O}), 1668(\mathrm{C}=\mathrm{O}) ;{ }^{1} \mathrm{H} \mathrm{NMR}(500 \mathrm{MHz}$, DMSO- $\left.d_{6}, \mathrm{ppm}\right): \delta 1.26\left(\mathrm{t}, 6 \mathrm{H}, 2 \mathrm{CH}_{3}\right), 2.48\left(\mathrm{~s}, 6 \mathrm{H}, 2 \mathrm{CH}_{3}\right)$, 3.92 (q, 4H, $2 \mathrm{CH}_{2}$ ), 6.76-7.90 (m, 12H, $8 \mathrm{Ph}-\mathrm{H}+4$ pyr-H), 8.45 (s, 2H, 2 pyr-5'-H), 9.18 (s, 2H, benzene-H); ${ }^{13} \mathrm{C}$ NMR (125 MHz, DMSO- $\left.d_{6}, \mathrm{ppm}\right): \delta 14.15,25.08,64.18,101.15$, $101.76,116.42$, 121.02, 122.88, 125.65, 128.78, 131.68, 135.24, $136.45,145.65,146.32,150.05,151.84,154.12,155.08,157.26$, 159.61, 162.74, 164.18, 165.46; MS, $m / z(\%): 1179\left[\mathrm{M}^{+}, 8\right]$, 156 [100, base peak]. Elemental analysis for $\mathrm{C}_{56} \mathrm{H}_{32} \mathrm{~N}_{10} \mathrm{O}_{8} \mathrm{~S}_{2} \mathrm{Cl}_{4}$ (1178.86): Calcd.: C, 57.06; H, 2.74; Cl, 12.03; N, 11.88; S, 5.44; Found: C, 57.00; H, 2.69; Cl, 11.98; N, 11.82; S, 5.40.

Compound 10a: Yield $55 \%$, m.p. $325-327^{\circ} \mathrm{C}$; IR (KBr, $\left.v_{\max }, \mathrm{cm}^{-1}\right): 1752(\mathrm{C}=\mathrm{O}), 1670(\mathrm{C}=\mathrm{O}) ;{ }^{1} \mathrm{H}$ NMR $(500 \mathrm{MHz}$, DMSO- $\left.d_{6}, \mathrm{ppm}\right): \delta 1.28\left(\mathrm{t}, 6 \mathrm{H}, 2 \mathrm{CH}_{3}\right), 2.45\left(\mathrm{~s}, 6 \mathrm{H}, 2 \mathrm{CH}_{3}\right)$, 3.83 (q, 4H, $2 \mathrm{CH}_{2}$ ), 6.78-7.90 (m, 12H, $8 \mathrm{Ph}-\mathrm{H}+4$ pyr-H),
8.52 (s, 2H, 2 pyr-5'-H), 8.62 (d, 4H, naphthalen-H); ${ }^{13} \mathrm{C} \mathrm{NMR}$ (125 MHz, DMSO- $\left.d_{6}, \mathrm{ppm}\right): \delta 14.08,25.12,64.26,101.15$, $101.72,116.36,120.42,121.45,123.10,128.94,131.65,135.22$, 136.38, 139.64, 145.65, 146.30, 149.80, 151.76, 154.22, 154.75, 157.18, 158.65, 159.64, 162.70, 165.44; MS, m/z (\%): 1196 $\left[\mathrm{M}^{+}, 18\right], 634$ [100, base peak]. Elemental analysis for $\mathrm{C}_{60} \mathrm{H}_{34} \mathrm{~N}_{10} \mathrm{O}_{8} \mathrm{~S}_{2} \mathrm{Cl}_{2} \mathrm{~F}_{2}$ (1196.01): C, 60.25; H, 2.87; Cl, 5.93; N, 11.71; S, 5.36; Found: C, 60.20; H, 2.80; Cl, 5.88; N, 11.66; S, 5.30 .

Compound 10b: Yield $56 \%$, m.p. $336-338{ }^{\circ} \mathrm{C}$; IR (KBr, $\left.v_{\max }, \mathrm{cm}^{-1}\right): 1746(\mathrm{C}=\mathrm{O}), 1674(\mathrm{C}=\mathrm{O}) ;{ }^{1} \mathrm{H}$ NMR $(500 \mathrm{MHz}$, DMSO- $\left.d_{6}, \mathrm{ppm}\right): \delta 1.30\left(\mathrm{t}, 6 \mathrm{H}, 2 \mathrm{CH}_{3}\right), 2.52\left(\mathrm{~s}, 6 \mathrm{H}, 2 \mathrm{CH}_{3}\right)$, 3.90 (q, 4H, $2 \mathrm{CH}_{2}$ ), 6.84-7.96 (m, 12H, $8 \mathrm{Ph}-\mathrm{H}+4$ pyr-H), 8.42 (s, 2H, 2 pyr-5'-H), 8.65 (d, 4H, naphthalen-H); ${ }^{13} \mathrm{C}$ NMR (125 MHz, DMSO- $\left.d_{6}, \mathrm{ppm}\right): \delta 14.38,25.12,64.24,101.23$, $101.66,116.53,121.10,121.78,122.80,128.70,131.65$, $135.34,136.48,139.05,145.72,146.40,150.12,151.80$, 154.16, 155.12, 157.34, 158.65, 159.78, 162.70, 165.40; MS, $\mathrm{m} / \mathrm{z}(\%): 1229\left[\mathrm{M}^{+}, 8\right], 165$ [100, base peak]. Elemental analysis for $\mathrm{C}_{60} \mathrm{H}_{34} \mathrm{~N}_{10} \mathrm{O}_{8} \mathrm{~S}_{2} \mathrm{Cl}_{4}$ (1228.92): C, 58.64; $\mathrm{H}, 2.79 ; \mathrm{Cl}, 11.54$; N, 11.40; S, 5.22; Found: calcd.: C, 58.60; H, 2.73; Cl, 11.50; N, 11.34; S, 5.16.

Biological assay: The antimicrobial activities were determined by Agar diffusion method. The microorganism inoculums were uniformly spread using sterile cotton swab on a sterile Petri dish containing Malt extract agar (for fungi) and nutrient agar (for bacteria). Each sample $(100 \mu \mathrm{L})$ was added to each well (6 mm diameter holes cut in the agar gel, $20 \mathrm{~mm}$ apart from one another). The systems were incubated for 24$48 \mathrm{~h}$ at $37{ }^{\circ} \mathrm{C}$ (for bacteria) and at $28{ }^{\circ} \mathrm{C}$ (for fungi). After incubation, microorganism growth was observed. Inhibition of the bacterial and fungal growth were measured in $\mathrm{mm}$. Tests were performed in triplicate ${ }^{33}$.

Pharmacology screening: All animals were obtained from Animal House Colony, Research Institute of Ophthalmology, Giza, Egypt.

Determination of acute toxicity $\left(\mathbf{L D}_{\mathbf{5 0}}\right)$ : The $\mathrm{LD}_{50}$ for compounds were determined by injected different gradual increased doses of the tested compounds to adult mail albino rats, then calculate the dose cause $50 \%$ animal death, according to Austen et al..$^{34}$ (Table-2).

Analgesic activity: Sixty Webster mice of both sexes weighting from $20-25 \mathrm{~g}$ were divided into 10 groups. One group was kept as control (received saline), the second group received vehicle (Gum acacia) and the third one received Valdecoxib $^{\circledR}$ as a reference drug, whereas the other groups received tested compounds (SC administration). Mice were dropped gently in a dry glass beaker of one-liter capacity maintained at $55-55.5{ }^{\circ} \mathrm{C}$. Normal reaction time in seconds for all animals was determined at time intervals of 10, 20,30, 45, 60, 90 and $120 \mathrm{~min}$. This is the interval extending from the instant the mouse reaches the hot beaker till the animals licks its feet or jump out of the beaker (dose $5 \mathrm{mg} / \mathrm{kg})^{35}$, relative potencies to valdecoxib ${ }^{\circledR}$ were determined (Table-3).

Antiparkinsonian activity: Groups of eight mail mice (18-20 g) were used. They were dosed orally with the tested compounds $\left(5 \mathrm{mg} / \mathrm{kg}\right.$ ) or the standard (benzatropine ${ }^{\circledR}, 5 \mathrm{mg}$ / $\mathrm{kg})^{36} 1 \mathrm{~h}$ prior to the administration of $0.5 \mathrm{mg} / \mathrm{kg}$ of oxotremorine ${ }^{\circledR}$ S.C. Rectal temperature was measured before 
administration of the compounds and $1 \mathrm{~h}$ after oxotremorine ${ }^{\circledR}$ dosage. The score for the recorded signs are zero (absent), one (slight), two (mediums) and three (highs) (Table-4).

\section{RESULTS AND DISCUSSION}

In the present work, 2-chloro-6-ethoxy-4-[(2-methyl-4oxo-9-(substituted phenyl)pyrido- $\left[3^{\prime}, 2^{\prime}: 4,5\right]$ thieno[3,2$d$ ] oxazin-7-yl]pyridines (2a,b) was used as starting material and it was prepared from the corresponding 1-(2-chloro-6ethoxypyridin-4-yl)-3-(substituted phenyl)prop-2-en-1-one $\mathbf{1 a}, \mathbf{b}$ according to our reported methods ${ }^{23,24}$ (Scheme-I).

Treatment of oxazinone derivatives $\mathbf{2 a , b}$ with aniline or phenylhydrazine in glacial acetic acid under reflux condition afforded the corresponding 7-(2-chloro-6-ethoxypyridin-4-yl)9-aryl-2-methyl-3-phenyl-pyrido[3', $\left.2^{\prime}: 4,5\right]$ thieno[3,2$d$ pyrimidine-4 $(3 H)$-one $(\mathbf{3 a}, \mathbf{b})$ and 7-(2-chloro-6-ethocypyridin-4-yl)-9-aryl-3-benzyl-2-methylpyrido [3', 2':4,5]thieno[3,2- $d$ ]pyrimidine-4(3H)-one $(\mathbf{4 a}, \mathbf{b})$, respectively. In addition, reacting of $\mathbf{2 a}, \mathbf{b}$ with hydrazine hydrate in refluxing ethanol afforded the corresponding $\mathrm{N}$-aminothienopyrimidine derivatives $(\mathbf{5 a}, \mathbf{b})$, which was reacted with phenylisothiocyanate to give the corresponding thiosemicarbazide derivatives $(\mathbf{6 a}, \mathbf{b})$, respectively (Scheme-II).

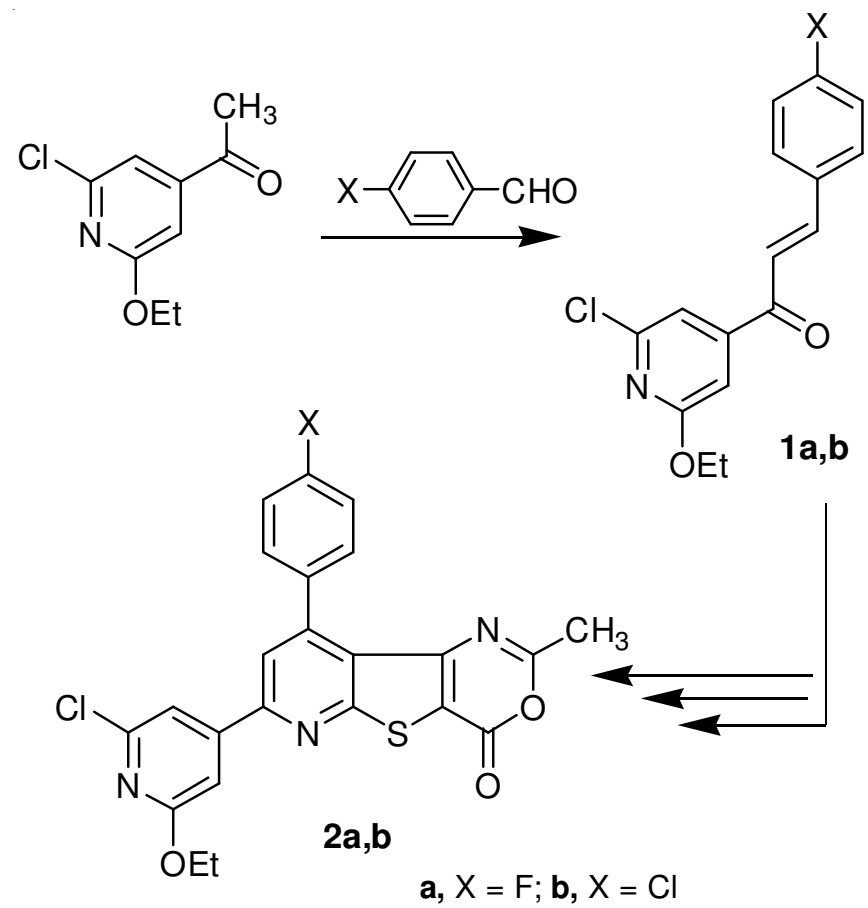

Scheme-I: Synthetic pathway for starting material 2<smiles>[X]c1ccc(-c2cc(-c3cc(Cl)nc(OCC)c3)nc3sc4c(=O)n(CC(C)N)c(C)nc4c23)cc1</smiles>

Scheme-II: Synthetic pathway for compounds 3-6 
Additionally, condensation of $\mathbf{5 a}, \mathbf{b}$ with $p$-fluorobenzaldehyde in refluxing ethanol containing a few drops of piperidine yielded the corresponding Schiff's bases 8a,b. Also, the reaction of $\mathbf{5 a}, \mathbf{b}$ with phthalic anhydride or 2,3,4,5-tetrachlorophthalic anhydride in refluxing acetic acid gave the corresponding 3-imido-pyrimidinone derivatives 7a-d (Scheme-III).

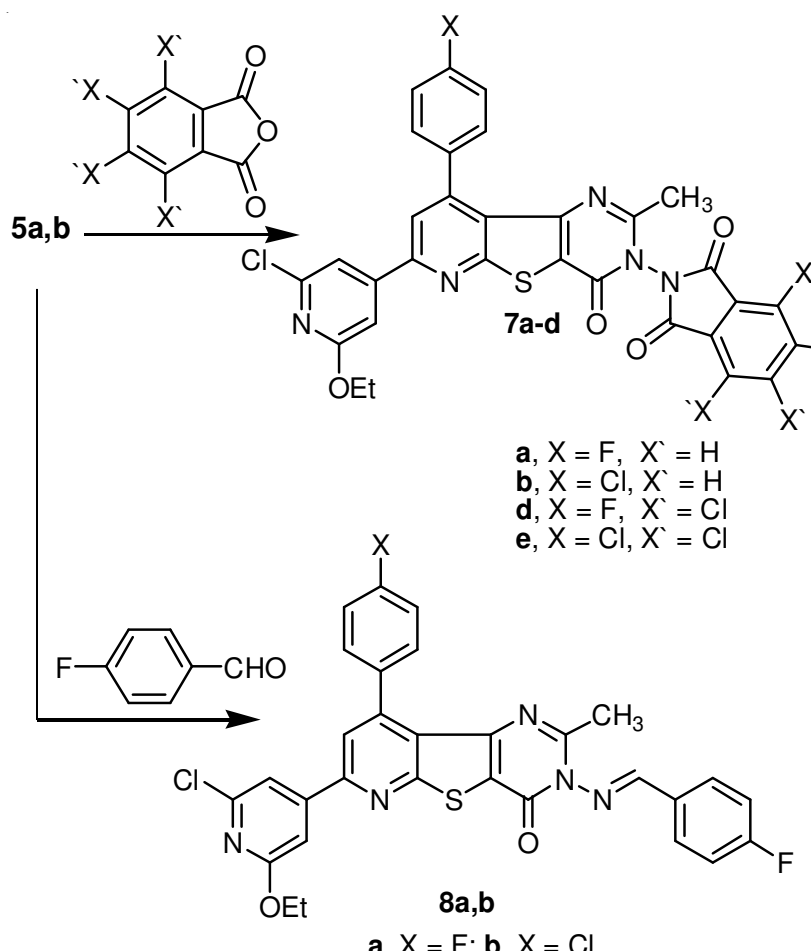

Scheme-III: Synthetic pathway for compounds $\mathbf{7}$ and $\mathbf{8}$

Finally, treatment of 5a,b with 1,2,4,5-benzenetetracarboxylic acid dianhydride or 1,8,4,5-naphthaline-tetracarboxylic acid dianhydride in acetic acid gave the corresponding biscompounds 9a,b and 10a,b, respectively (Scheme-IV).
Antimicrobial activity: in vitro Antimicrobial screening of compounds prepared in this study was carried out using cultures of four fungal strains, including Aspergillus niger and Candida albicans as well as four bacteria species, namely, Gram positive bacteria, Staphylococcus aureus and Bacillus subtilis, Gram negative bacteria, Escherichia coli. Streptomycin and fusidic acid were used as positive reference drugs to evaluate the potency of the tested compounds under the same conditions. Most of the newly synthesized compounds showed highest with respect to control drugs. Ten of the synthesized compounds 3a, 3b, 4b, 6a, 6b, 7c, 7d, 8a, 9b and 10b exhibited potent antibacterial and antifungal bioactivity compared with the standard drug used. The other tested compounds were found to exhibit a moderate to low antibacterial activities (Table-1). The most active compound is 8a which due to the increase in the fluorine number in the phenyl rings. The results of antimicrobial activities were shown in Table-1.

Pharmacological screening: Initially, the acute toxicity of the compounds was assayed via the determination of their $\mathrm{LD}_{50}$ (Table-2). The tested two pharmacological activities namely,

\begin{tabular}{cc} 
TABLE-2 \\
ACUTE TOXICITY LD \\
AND STARTING COMPOUNTHESIZED \\
\hline \multicolumn{2}{c}{ ANDS } \\
\hline Compound No. & LD $_{50}\left(\mathrm{mg} \mathrm{kg}^{-1}\right)$ \\
\hline Valdecoxib $^{\circledR}$ & 1.68 \\
$\mathbf{3 b}$ & 1.75 \\
$\mathbf{3 a}$ & 1.81 \\
$\mathbf{4 a}$ & 2.35 \\
$\mathbf{6 a}$ & 2.30 \\
$\mathbf{6 b}$ & 1.96 \\
$\mathbf{7 c}$ & 2.48 \\
$\mathbf{7 d}$ & 2.82 \\
$\mathbf{8 a}$ & 3.54 \\
$\mathbf{9 b}$ & 1.72 \\
$\mathbf{1 0 b}$ & 2.40 \\
\hline
\end{tabular}

TABLE-1

ANTIMICROBIAL ACTIVITIES OF NEWLY SYNTHESIZED COMPOUNDS 3-10

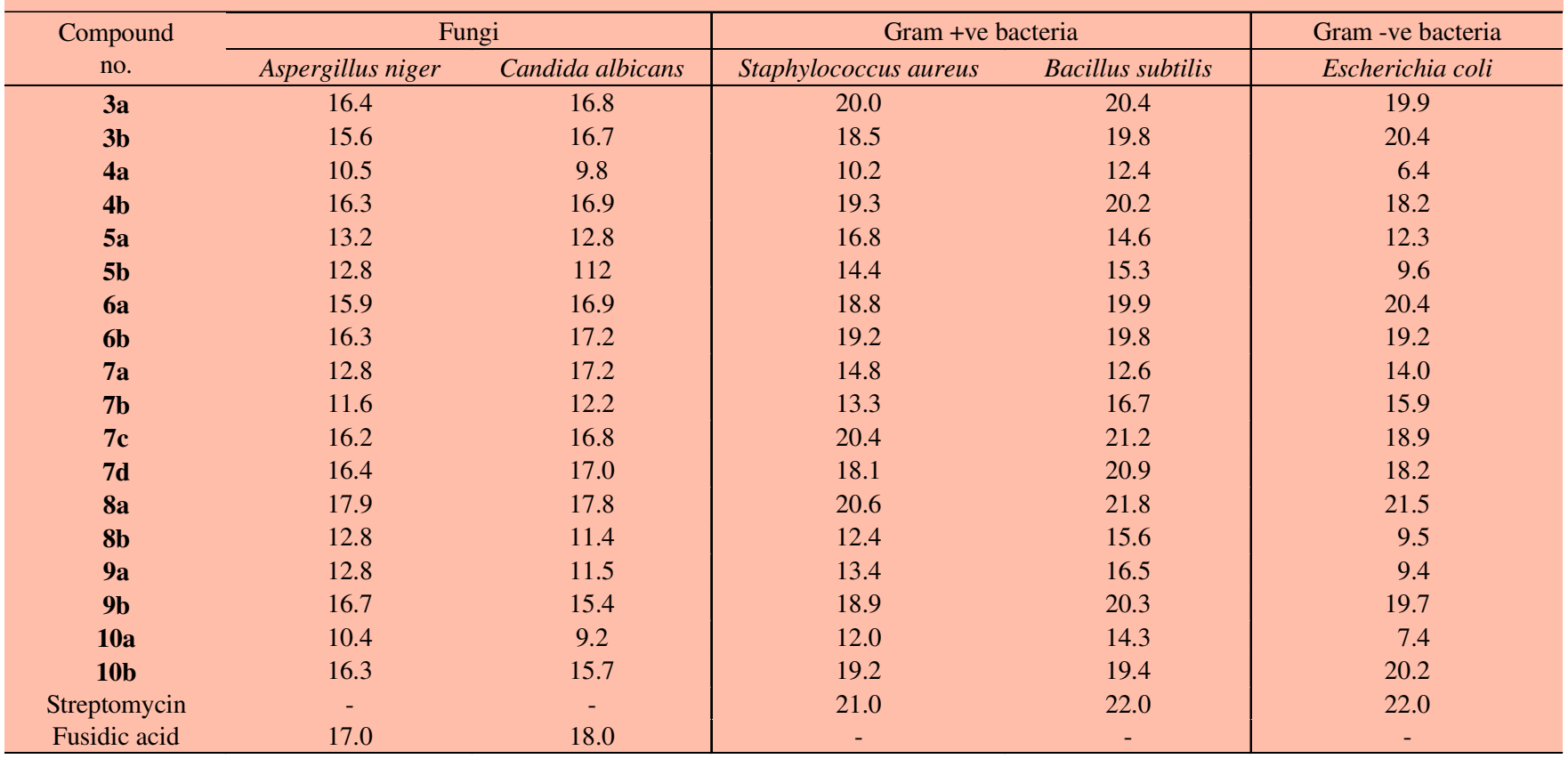




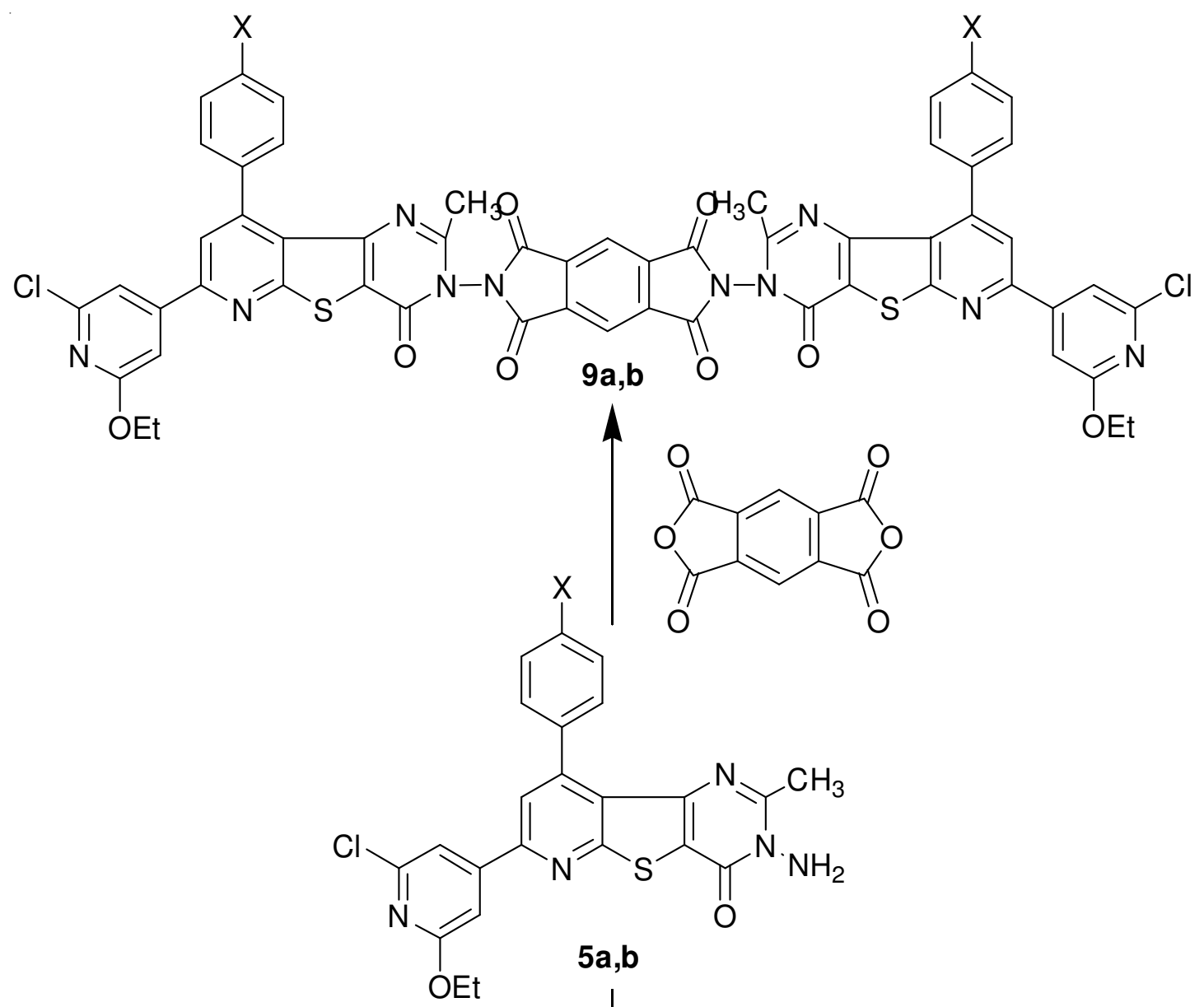

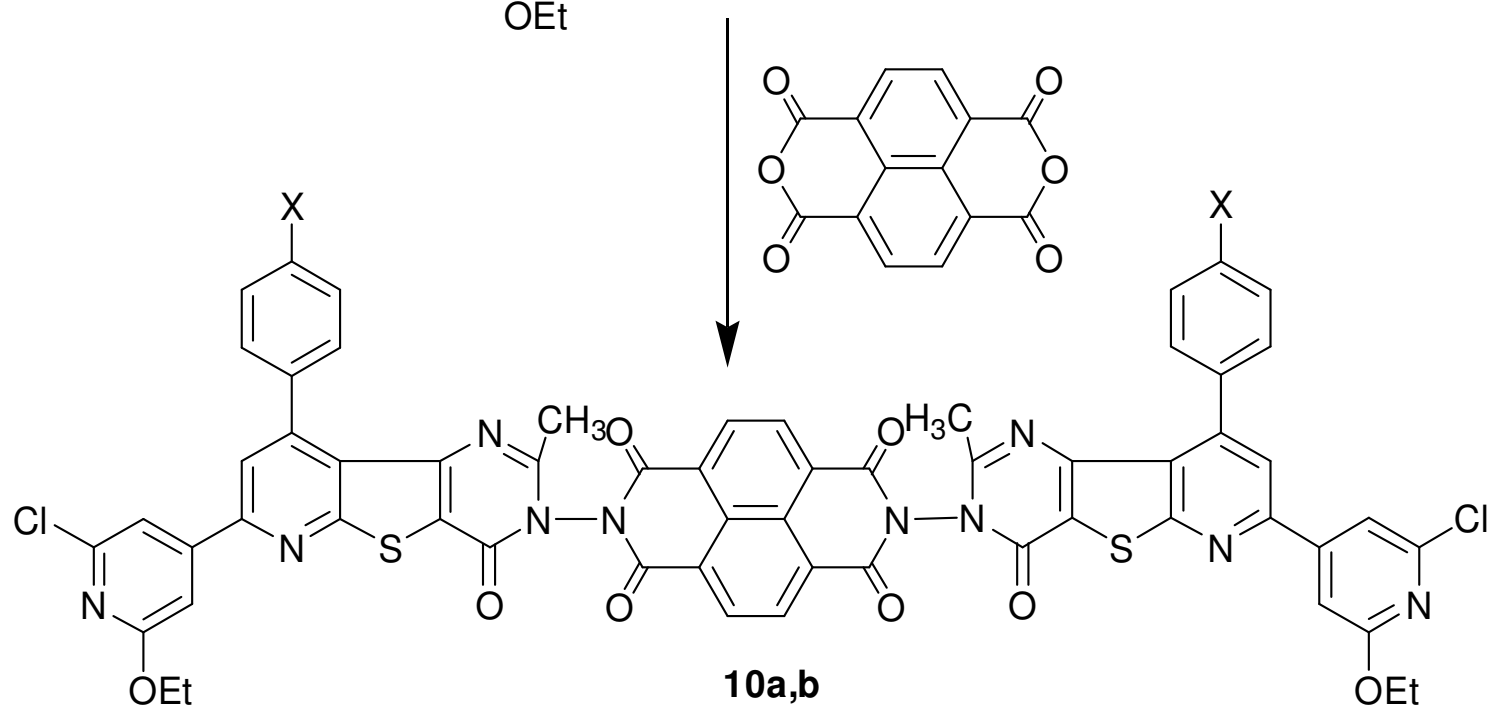

$\mathbf{a}, \mathrm{X}=\mathrm{F} ; \mathbf{b}, \mathrm{X}=\mathrm{Cl}$

Scheme-IV: Synthetic pathway for compounds $\mathbf{9}$ and $\mathbf{1 0}$

analgesic and antiparkinsonian activities of their different biological receptors yet all of a neurological. Ten representative compounds $\mathbf{3 a}, \mathbf{b}, \mathbf{4 a}, \mathbf{6 a}, \mathbf{b}, \mathbf{7 c}, \mathbf{d}, \mathbf{8 a}, \mathbf{9 b}, \mathbf{1 0 b}$ were studied with respect to analgesic and antiparkinsonian activities.

Analgesic activity: All compounds tested exhibited analgesic activities in a hot plate assay (Table-3). The most potent are compounds 7c and 8a showed higher activity than voldecoxib ${ }^{\circledR}$ by nearly 140-160\% (compound $\mathbf{8 a}$ showed the most pronounced effect). Also, the analgesic activities of another compounds approached those of valdecoxib ${ }^{\circledR}$ and showed $62-84 \%$ activity as compared to valdecoxib ${ }^{\circledR}(=100 \%)$ (Table-3).

Antiparkinsonian activity: The antiparkinsonian activity measured by the ability of compounds to protect animals against the parkinsonian like sings induced by agonists. Compounds 3a, 4a, 6a and showed nearly no antiparkinsonian activities, while compounds $\mathbf{3 b}, \mathbf{6 b}$ and $\mathbf{7 d}$ showed moderate antiparkinsonian activities. Compounds 7c, 8a and 10a are the most potent antiparkinsonian agents (Table-4). 


\begin{tabular}{|c|c|c|c|c|c|c|c|}
\hline \multirow[b]{3}{*}{ Comp. No. } & \multicolumn{6}{|c|}{$\begin{array}{c}\text { TABLE-3 } \\
\text { ANALGESIC ACTIVITIES OF SELECTED COMPOUNDS IN A HOT PLATE ASSAY }\end{array}$} & \\
\hline & \multicolumn{7}{|c|}{ Analgesic activity related to Valdecoxib $^{\circledR}$ after } \\
\hline & $\begin{array}{l}10 \mathrm{~min} \\
\pm \mathrm{SE}\end{array}$ & $\begin{array}{l}20 \mathrm{~min} \\
\pm \mathrm{SE}\end{array}$ & $\begin{array}{l}30 \mathrm{~min} \\
\pm \mathrm{SE}\end{array}$ & $\begin{array}{l}45 \mathrm{~min} \\
\pm \mathrm{SE}\end{array}$ & $\begin{array}{l}60 \mathrm{~min} \\
\pm \mathrm{SE}\end{array}$ & $\begin{array}{l}90 \min \\
\pm \mathrm{SE}\end{array}$ & $\begin{array}{l}120 \mathrm{~min} \\
\pm \mathrm{SE}\end{array}$ \\
\hline Valdecoxib $_{\circledast}$ & $1.00 \pm 0.010$ & $1.00 \pm 0.010$ & $1.00 \pm 0.010$ & $1.00 \pm 0.010$ & $1.00 \pm 0.010$ & $1.00 \pm 0.010$ & $1.00 \pm 0.010$ \\
\hline $\mathbf{3 a}$ & $0.79 \pm 0.010$ & $0.85 \pm 0.013$ & $0.86 \pm 0.013$ & $0.88 \pm 0.014$ & $0.86 \pm 0.015$ & $0.86 \pm 0.010$ & $0.85 \pm 0.017$ \\
\hline $3 \mathbf{b}$ & $0.84 \pm 0.015$ & $0.94 \pm 0.014$ & $0.92 \pm 0.017$ & $0.97 \pm 0.021$ & $0.96 \pm 0.030$ & $0.94 \pm 0.015$ & $0.95 \pm 0.024$ \\
\hline $4 \mathbf{a}$ & $0.93 \pm 0.010$ & $0.94 \pm 0.010$ & $0.93 \pm 0.015$ & $0.90 \pm 0.019$ & $0.85 \pm 0.021$ & $0.82 \pm 0.016$ & $0.65 \pm 0.012$ \\
\hline $6 a$ & $0.63 \pm 0.014$ & $0.75 \pm 0.010$ & $0.82 \pm 0.001$ & $0.84 \pm 0.014$ & $0.87 \pm 0.015$ & $0.86 \pm 0.013$ & $0.85 \pm 0.035$ \\
\hline $6 \mathbf{b}$ & $0.66 \pm 0.010$ & $0.66 \pm 0.016$ & $0.75 \pm 0.014$ & $0.74 \pm 0.016$ & $0.76 \pm 0.016$ & $0.76 \pm 0.015$ & $0.79 \pm 0.014$ \\
\hline $7 c$ & $0.98 \pm 0.013$ & $0.99 \pm 0.012$ & $1.42 \pm 0.135$ & $1.58 \pm 0.211$ & $1.55 \pm 0.351$ & $1.58 \pm 0.341$ & $1.45 \pm 0.451$ \\
\hline 7d & $0.65 \pm 0.011$ & $0.64 \pm 0.011$ & $0.88 \pm 0.011$ & $0.89 \pm 0.016$ & $0.90 \pm 0.021$ & $0.90 \pm 0.017$ & $0.89 \pm 0.018$ \\
\hline $8 \mathbf{a}$ & $1.30 \pm 0.176$ & $1.44 \pm 0.14$ & $1.46 \pm 0.131$ & $1.45 \pm 0.201$ & $1.42 \pm 0.321$ & $1.40 \pm 0.291$ & $1.41 \pm 0.278$ \\
\hline $9 b$ & $0.60 \pm 0.011$ & $0.67 \pm 0.012$ & $0.75 \pm 0.013$ & $0.76 \pm 0.019$ & $0.78 \pm 0.014$ & $0.79 \pm 0.012$ & $0.78 \pm 0.012$ \\
\hline $10 \mathrm{~b}$ & $0.89 \pm 0.011$ & $0.88 \pm 0.010$ & $0.90 \pm 0.010$ & $0.92 \pm 0.016$ & $0.93 \pm 0.015$ & $0.92 \pm 0.014$ & $0.90 \pm 0.016$ \\
\hline
\end{tabular}

TABLE-4

ANTIPARKINSONIAN ACTIVITIES OF SYNTHESIZED COMPOUNDS AS COMPARED WITH BENZOTROPENE ${ }^{\circledR}$

\begin{tabular}{ccccc}
\hline $\begin{array}{c}\text { Compound } \\
\text { no. }\end{array}$ & $\begin{array}{c}\text { Salivation and } \\
\text { lacrimation score }\end{array}$ & $\begin{array}{c}\text { Tremors } \\
\text { score }\end{array}$ & $\begin{array}{c}\text { \% Decrease from Oxotremerine } \\
\text { rectal temperature }(\%) \pm \text { SE }\end{array}$ & $\begin{array}{c}\text { Relative potency compared to } \\
\text { Benzotropene mesilate } \pm \text { SE }\end{array}$ \\
\hline Control & 0 & 0 & 0 & 0 \\
Benzotropene & 1 & 1 & $25.0 \pm 0.400$ & $1.00 \pm 0.09$ \\
3a & 3 & 3 & $4.0 \pm 0.010$ & $0.13 \pm 0.014$ \\
$\mathbf{3 b}$ & 2 & 2 & $16.0 \pm 0.288$ & $0.59 \pm 0.060$ \\
$\mathbf{4 a}$ & 3 & 3 & $5.0 \pm 0.011$ & $0.15 \pm 0.015$ \\
$\mathbf{6 a}$ & 3 & 3 & $4.0 \pm 0.016$ & $0.13 \pm 0.012$ \\
$\mathbf{6 b}$ & 1 & 1 & $17.0 \pm 0.301$ & $0.65 \pm 0.07$ \\
$\mathbf{7 c}$ & 1 & 1 & $20.0 \pm 0.142$ & $0.82 \pm 0.08$ \\
$\mathbf{7 d}$ & 2 & 2 & $11.0 \pm 0.101$ & $0.41 \pm 0.030$ \\
$\mathbf{8 a}$ & 1 & 1 & $21.0 \pm 0.368$ & $0.80 \pm 0.076$ \\
$\mathbf{9 a}$ & 3 & 3 & $5.0 \pm 0.010$ & $0.17 \pm 0.010$ \\
$\mathbf{1 0 a}$ & 1 & 1 & $21.0 \pm 0.481$ & $0.80 \pm 0.070$ \\
\hline
\end{tabular}

\section{Conclusion}

In conclusion, we reported herein antimicrobial activities of some new synthesized methyl-pyrido[3',2':4,5] thieno[3,2d]-pyrimidine derivatives (3-10) by using 2-chloro-6-ethoxy4-[(2-methyl-4-oxo-9-(substituted phenyl)pyrido[3',2':4,5]thieno[3,2-d] oxazin-7-yl]pyridines $\mathbf{2 a}, \mathbf{b}$ as starting materials. All the newly synthesized compounds were evaluated for antimicrobial activities. Ten of the synthesized compounds $\mathbf{3 a}$, 3b, 4b, 6a, 6b, 7c, 7d, 8a, 9b and 10b exhibited potent antibacterial and antifungal bioactivity compared with the standard drug used. The other tested compounds were found to exhibit a moderate to low antibacterial activities (Table-1). The most active compound is $\mathbf{8} \mathbf{a}$ which due to the increase in the fluorine number in the phenyl rings.

\section{ACKNOWLEDGEMENTS}

The kind helps of Dr. M.M. Abdalla, Research Unit, HiCare, Pharmaceutical Co., Cairo, Egypt, for carrying out the pharmacological screening are acknowledged. The authors extend their appreciation to the Deanship of Scientific Research at King Saud University for funding the work through the research group project No. RGP-VPP-0172.

\section{REFERENCES}

1. G. Steiner, W. Lubisch, A. Bach, F. Emling, K. Wicke, H.J. Teschendorf, B. Behl, F. Kerrigan and S. Cheetham, German Patent 19636769 (1996); Chem. Abstr., 128, 217381 (1998).

2. M. Modica, M. Santagati, A. Santagati, V. Cutuli, N. Mangano and A. Caruso, Pharmazie, 55, 500 (2000).

3. M.M. El-Kerdawy, M.Y. Yousif, A.A. El-Emam, M.A. Moustafa and M.A. El-Sherbeny, Boll. Chim. Farm., 135, 301 (1996).

4. K. Eichenberger, P. Schmidt and E. Schweizer, German Patent 1937459 , (1969); Chem. Abstr., 72, 100743 (1970).

5. A. Kling, U.E.W. Lange, H. Mack, M.H.M. Bakker, K.U. Drescher, W. Hornberger, C.W. Hutchins, A. Möller, R. Müller, M. Schmidt, L. Unger, K. Wicke, K. Schellhaas and G. Steiner, Bioorg. Med. Chem. Lett., 15, 5567 (2005).

6. R.V. Chambhare, B.G. Khadse, A.S. Bobde and R.H. Bahekar, Eur. J. Med. Chem., 38, 89 (2003).

7. N.A. Santagati, A. Caruso, V.M. Cutuli and F. Caccamo, IL Farmaco, 50, 689 (1995).

8. L.D. Jennings, S.L. Kincaid, Y.D. Wang, G. Krishnamurthy, C.F. Beyer, J.P. McGinnis, M. Miranda, C.M. Discafani and S.K. Rabindran, Bioorg. Med. Chem. Lett., 15, 4731 (2005).

9. M.D. Meyer, R.J. Altenbach, F.Z. Basha, W.A. Carroll, S. Condon, S.W. Elmore, J.F. Kerwin, K.B. Sippy, K. Tietje, M.D. Wendt, A.A. Hancock, M.E. Brune, S.A. Buckner and I. Drizin, J. Med. Chem., 43, 1586 (2000).

10. S.S. Laddha, S.G. Wadokar and S.K. Meghal, ARKIVOC, 1 (2006).

11. P.N. Bhargava and M.R. Chaurasia, J. Med. Chem., 11, 404 (1968).

12. S.S. Parmar and R. Kumar, J. Med. Chem., 11, 635 (1968). 
13. V. Alagarsamy, U.S. Pathak, R. Venkateshperumul, et al., Indian. J. Pharm. Sci., 65, 293 (2003).

14. E.B. Skibo, X. Huang, R. Martinez, R.H. Lemus, W.A. Craigo and R.T. Dorr, J. Med. Chem., 45, 5543 (2002).

15. A. Pandey, D.L. Volkots, J.M. Seroogy, J.W. Rose, J.-C. Yu, J.L. Lambing, A. Hutchaleelaha, S.J. Hollenbach, K. Abe, N.A. Giese and R.M. Scarborough, J. Med. Chem., 45, 3772 (2002).

16. A.S. Oganisyan, A.S. Noravyan, I.A. Dzhagatspanyan and A.G. Akopyan, Pharm. Chem. J., 35, 127 (2001).

17. B.V. Ashalatha, B. Narayana, K.K. Vijaya Raj and N. Suchetha Kumari, Eur. J. Med. Chem., 42, 719 (2007).

18. S. Selleri, F. Bruni, A. Costanzo, G. Guerrini, P. Malmberg Aiello, G. Iavarone and C. Martini, Eur. J. Med. Chem., 27, 985 (1992).

19. M. Kabak, A. Elmali and Y. Elerman, J. Mol. Struct., 477, 151 (1999).

20. P.R. Patel, B.T. Thaker and S. Zele, Indian J. Chem., 38A, 563 (1999).

21. P. Pattanayak, R. Sharma and P.K. Sahoo, Med. Chem. Res., 18, 351 (2009).

22. S.K. Gupta, P.K. Sharma, M. Bansal and B. Kumar, E-J. Chem., 8, 594 (2011)

23. A.S.M. Hossan, H.M.A. Abu-Melha, M.A. Al-Omar and A.E. Amr, Molecules, 17, 13642 (2012)

24. A.A. Fayed, A. El-Galil E. Amr, M.A. Al-Omar and E.E. Mostafa, Russ. J. Bioorg. Chem., 40, 308 (2014).
25. A.M. Al-Mohizea, M.A. Al-Omar, M.M. Abdalla and A.E. Amr, Int. J. Biol. Macromol., 50, 171 (2012).

26. N.O. Al-Harbi, S.A. Bahashwan, A.A. Fayed, M.S. Aboonq and A.E. Amr, Int. J. Biol. Macromol., 57, 165 (2013).

27. S.A. Bahashwan, N.O. Al-Harbi, A.A. Fayed, A.E. Amr, K.A. Shadid, A.M. Alalawi and I.M.S. Bassati, Int. J. Biol. Macromol., 51, 7 (2012).

28. N.H. Ouf and A.E. Amr, Monatsh. Chem., 139, 579 (2008).

29. A.E. Amr, A.M. Mohamed, S.F. Mohamed, N.A. Abdel-Hafez and A.G. Hammam, Bioorg. Med. Chem., 14, 5481 (2006).

30. A.-G.E. Amr, M.I. Hegab, A.A. Ibrahiem and M.M. Abdulla, Monatsh. Chem., 134, 1395 (2003).

31. A.-G.E. Amr, J. World Chem., 4, 201 (2009).

32. S.F. Mohamed, E.M. Flefel, A.E. Amr and D.N. Abd El-Shafy, Eur. J. Med. Chem., 45, 1494 (2010).

33. J.A. Smania Jr., F.D. Monache, E.F.A. Smania and R.S. Cuneo, Int. J. Med. Mushrooms, 1, 325 (1999).

34. K.F. Austen and W.E. Brocklehurst, J. Exp. Med., 113, 521 (1961).

35. A. Tgolsen, G.H. Rofland, O.G. Berge and K. Hole, J. Pharmacol. Ther., 25, 241 (1991).

36. M.D. Tyahr, The Basal Ganginia, Raven Press New York, p. 293 (1976). 